\title{
The Galaxy Distribution as a Voronoi Foam
}

\author{
Vincent Icke and Rien van de Weygaert \\ Sterrewacht Leiden, Postbus 9513, 2300 RA Leiden, The Netherlands
}

(Received I990 December I0; in original form I990 May 3)

\section{SUMMARY}

We present a précis of a description of the large-scale galaxy distribution in the Universe. It is based on the finding that underdense regions in the cosmic mass distribution expand with respect to the background and become more and more spherical. This physical mechanism produces a geometrical model, which we have called Voronoi foam, for the asymptotic distribution ('skeleton') of the cosmic mass on IO-200 Mpc scales. Voronoi foam is a packing of polyhedral cells uniquely determined by the sites of the initial underdense regions. The walls are identified as the pancakes, the edges as the filaments, and the vertices as the clusters in the galaxy distribution.

The Voronoi tessellation is a good model for the large-scale galaxy distribution. It does not describe that distribution on small scales, but it forms a useful complement to N-body techniques, which have a low dynamic range and which suffer from sampling noise and boundary effects on very large scales. The Voronoi model enables one to study, in arbitrary detail, what the consequences of a cellular galaxy distribution are; we consider in particular the cluster distribution and the appearance of deep pencil-beam surveys.

Probably the most remarkable result of this description is the fact that the correlation function of the vertices in the Voronoi tessellation is a power law with a slope and amplitude totally in accordance with the observed cluster-cluster correlation, indicating that this function could be due to cellular geometry of the galaxy distribution on large scales, rather than to the dynamics of these structures.

\section{INTRODUCTION}

On very large scales (on the order of the particle horizon, i.e. some $7 \mathrm{Gpc}$ ) the Universe is well understood in the theoretical framework of the Friedmann-Robertson-Walker (FRW) solutions of the Einstein equations. This is most clearly shown by the near-perfect isotropy and black body spectrum of the microwave background radiation. Deviations from isotropy, which would be the most direct link to the origin of structure in the Universe, have not yet been detected. In fact, the upper limits on the anisotropies are so small that there seems to be a discrepancy with the graininess of the visible baryon distribution. On small scales (on the order of small groups of galaxies, up to I megaparsec or so) one expects that strongly non-linear evolution and dissipative effects play an important role. This makes it very difficult to extract direct information on the evolution of structures on these scales.

On scales between 10 and $500 \mathrm{Mpc}$, the structure has evolved only mildly since the recombination epoch. Hence, the mass and velocity distributions on these scales are very suitable for studying the formation of such structure (see Oort 1983, for a review). 

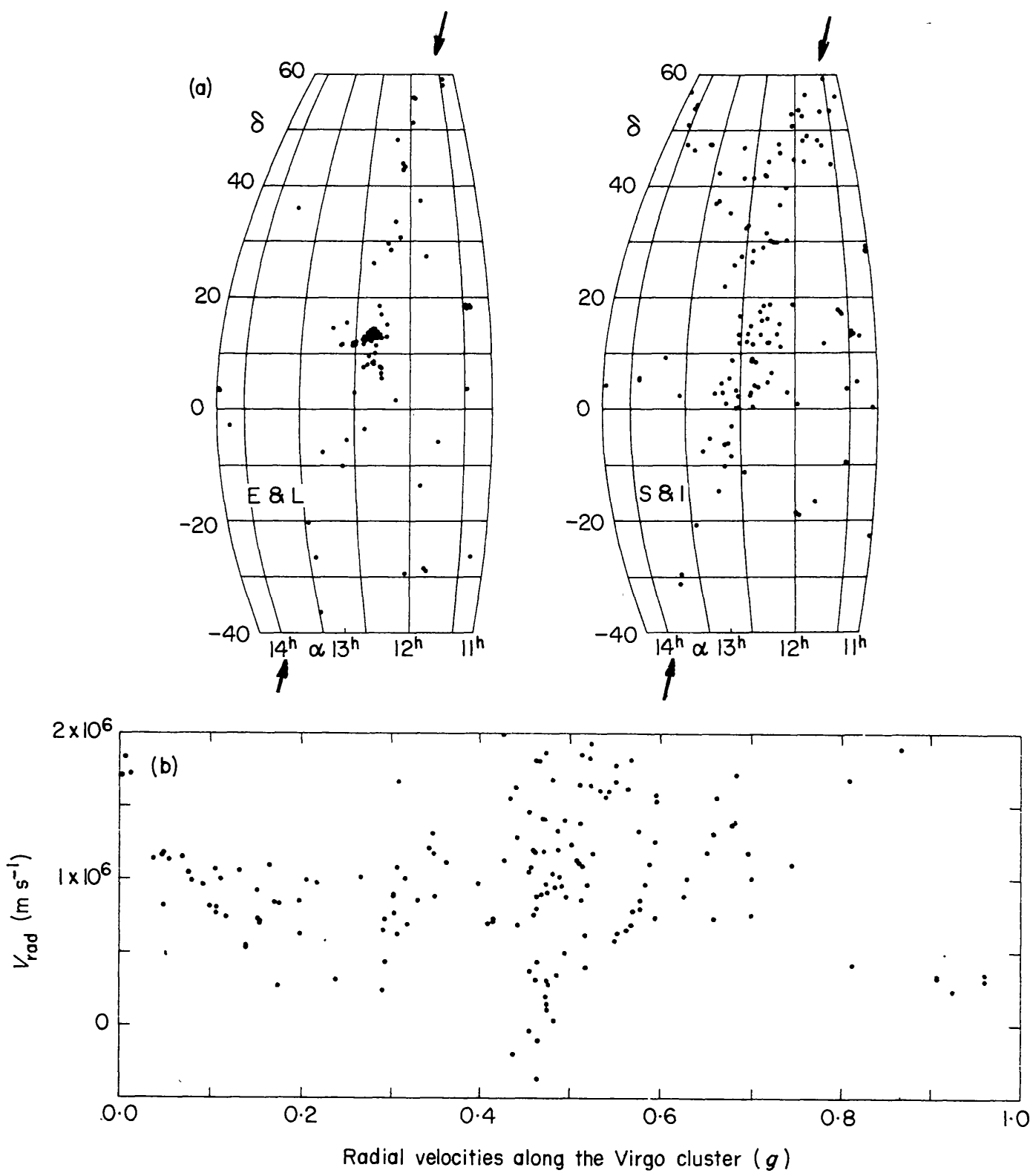

FIG. I. (a) A slice of the Universe in the constellation Virgo: left, elliptical and lenticular galaxies; right, spiral and irregular galaxies. (b) Radial velocities of these galaxies, as a function of their position along the major axis of the cluster (indicated by the arrows). Today's larger scale surveys usually show such data in the form of piewedge diagrams. After Icke (1972).

The last two decades have brought a steadily improving collection of observations of the properties of the large-scale structure (e.g. Kirshner et al. I98I, I987; Oort 1983; Giovanelli, Haynes \& Chincarini 1986; De Lapparent, Geller \& Huchra 1986; Geller \& Huchra 1989). We are even getting an idea of the non-Hubble motions on these scales (Dressler et al. 1987; Lynden-Bell et al. 1988), providing us with a potentially unbiased tracer of the mass distribution (e.g. Strauss \& Davis 1989; Yahil I989; Dekel, Bertschinger \& Faber 1990; Bertschinger et al. 1990). 


\section{SOME HISTORY}

The observations of large-scale structure date back to the work of Shapley \& Ames (1932), whose catalogue of bright stellar systems showed the dramatic excess of galaxies in some regions of the sky, notably the Virgo Cluster (see Oort 1983). Even in those early days, it was evident that the distribution of luminous matter on large scales is distinctly non-Poissonian. But until about 1970 , this remarkable fact was hardly a subject of systematic study, even though the catalogue of galaxy positions and redshifts compiled by Humason, Mayall \& Sandage (1956) contained a wealth of evidence about the curious structure of the nearby Universe. The clustering first studied by Abell (1958), and most apparent in the galaxy counts by Shane \& Wirtanen (I967), was thought to be analogous to that which is observed in open clusters and associations of galactic stars.

Interpretation of the non-Poissonian galaxy distribution was initiated by Rubin (I954) and Limber (I953, I954), who introduced the two-point correlation function, and by Neymann \& Scott (1955), who used the angular autocorrelation function of the Lick survey counts, but it was not until Totsuji \& Kihara (1969) and Peebles (1980, and references therein) that the two-point correlation function of galaxies was reliably estimated.

Oort (1970) emphasized the importance of larger structures because these, due to their long evolutionary time-scales, can be expected to give information about the earliest times of structure formation. In keeping with this, Icke (1972, 1973) hypothesized that such large aggregates (at least as large as I5 Mpc) form before galaxies (a scenario which has been pioneered by Zel'dovich and his co-workers in the context of the 'pancake' picture of galaxy formation), and he tried to find evidence for coherent objects on a scale of $15^{-20} \mathrm{Mpc}$ by studying the distribution of galaxies in positionvelocity space, a technique that is now known as 'slicing' (Fig. I).

Icke's efforts to corroborate his hypothesis were largely unsuccessful, due to a conspicuous lack of good observations. Appreciable improvements of the data by Humason, Mayall \& Sandage (1956) were not published until the beginning of the I980s, when several extensive galaxy redshift surveys became available (Sandage \& Tammann I98I ; Fisher \& Tully I98I ; Huchra et al. 1983). These redshift surveys provided evidence for the early suggestion by Einasto (1978; see also Einasto, Joêveer \& Saar I980) of cell-like structures in the distribution of galaxies. The mapping of, e.g. the Local Supercluster (Tully I982), the Coma Supercluster (Gregory \& Thompson 1978), and the enormous $140 \mathrm{Mpc}$ long chain of galaxies forming the Perseus Supercluster (Giovanelli, Haynes \& Chincarini 1986) showed that galaxies cluster in pancake-like and filamentary structures, while the redshift surveys also revealed the existence of voids in the distribution of galaxies (Gregory \& Thompson 1978; Einasto, Joêveer \& Saar 1980; Davis et al. 1982; Kirshner et al. I98I, I987).

These voids are enormous regions, tens of megaparsecs in extent, wherein few or no galaxies are found. In particular the discovery of the Boötes void (Kirshner et al. I98I) made clear how large these voids can be. Since 1986 the publication of new redshift slices from the second CfA redshift survey (De Lapparent, Geller \& Huchra I986; see also Da Costa \& Pellegrini I988 for 


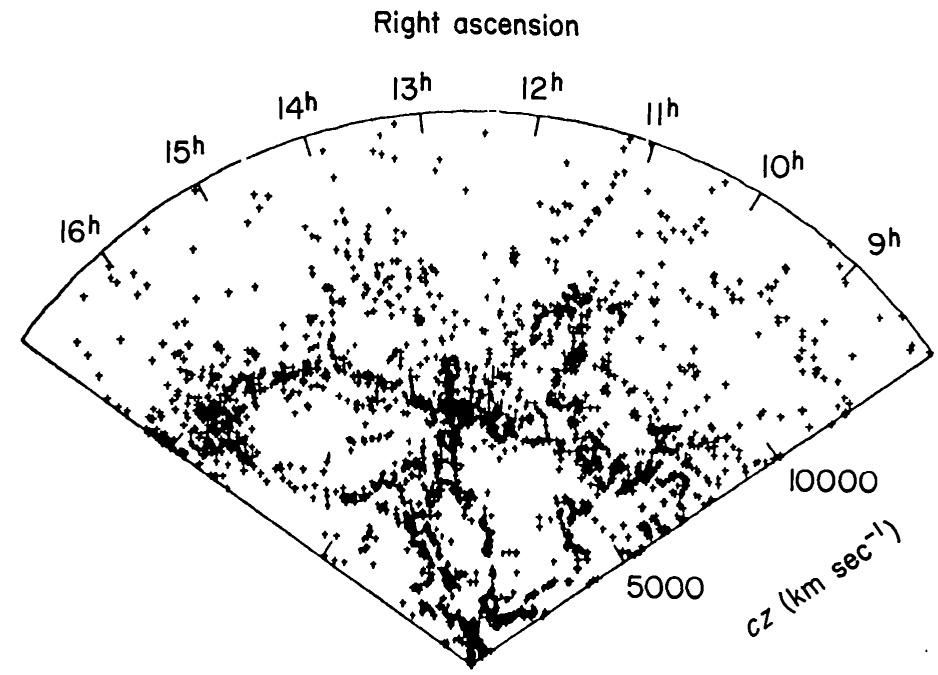

FIG. 2. A combination of CfA redshift survey slices; reproduced from Geller \& Huchra (1989), with the kind permission of the American Association for the Advancement of Science (AAAS). See also De Lapparent, Geller \& Huchra (I986).

the southern sky) confirmed the existence of a cellular or sponge-like arrangement of galaxies. The recent discovery by the CfA group (Geller \& Huchra 1989) of a $60 \times 170 \times 5 h^{-1} \mathrm{Mpc}$ 'Great Wall' of galaxies, and the discovery by Broadhurst et al. (1990) of a regular 'spiky' redshift distribution of galaxies in a narrow pencil-beam survey along the South and North galactic poles, are suggestive of cellular structures on scales as large as IOO Mpc.

The general impression from all these observations is a Universe in which the galaxies are situated in walls (pancakes), denser filaments, and very dense nodes, forming a network which surrounds huge voids (Fig. 2). Further details about the observed properties of the large-scale galaxy distribution can be found in the reviews by Oort (I983), Geller (I988), Bahcall (I988) and Rood (1988).

\section{PHYSICAL THEORY: SPOTLIGHT ON VOIDS}

Most theories of the formation of structure on large scales are based on the gravitational instability scenario (e.g. Peebles I980). Comprehensive reviews treating these theories were presented by Jones (1976), Efstathiou \& Silk (1983), Szalay (I988), Shandarin \& Zel'dovich (1989), Bond (1990), and Efstathiou (1990). Peebles \& Silk (1990) have recently tested the five most popular theories against a number of observational constraints. Although no firm conclusions were reached, they find that either cold dark matter plus inflation or a low-density Universe with baryonic dark matter are slightly favoured.

The description of the large-scale structure which we are about to present exploits the notion that voids play an essential dynamical role in the formation of such structure. This description, which we have called 'Voronoi foam', is an approximation of clustering on large scales based on the assumption that the dominant physical mechanism is Newtonian pressure- 
free gravity. We will show that this leads to a matter distribution which, in an asymptotic sense, forms a unique partitioning of space called a Voronoi tessellation.

On scales comparable with the particle horizon, the formation of structure must be due to perturbations of a FRW-type universe (Lifschitz I946), which is analytically difficult and not securely linked to observations. On small scales, galaxy formation is dominated by dissipative processes and is theoretically still quite intractable. But on intermediate scales, Newtonian gravitational instability should suffice for describing the formation of structure. Hydrodynamical details such as pressure effects are unlikely to be important in the progenitors of structures in the $10-500 \mathrm{Mpc}$ regime, so we can restrict ourselves to a 'dust' equation of state. The potential $\Phi$ near any point $(x, y, z)$ of a self-gravitating medium can be written as

$$
\Phi=\sum_{i j k} a_{i j k} x^{i} y^{j} z^{k}
$$

Near a density maximum, the leading terms are the quadratic ones, which, by a suitable orientation of Cartesian coordinates, can be written as

$$
\Phi=A x^{2}+B y^{2}+C z^{2}+\ldots
$$

Neglecting terms of higher than second order, this is the potential of a homogeneous ellipsoid. That should be no surprise: the smallest closed contours in any topographical map are ellipses.

The collapse of high-density regions can thus be approximated by considering the homologous motion of ellipsoids. An early treatment of this problem was given by Zel'dovich (1965), who considered the analogy between the evolution of a Newtonian homogeneous spheroid with anisotropic linear velocity and of a section of an anisotropic homogeneous cosmological model in the general theory of relativity. The equations of motion in both cases have the same form; the tidal field is determined by the shape of the surface of the ellipsoid in the first case, and by the topology of the model in the second. However, the generic properties of collapsing or expanding structures in Newtonian gravitating media are not always true in general relativity (Barrow I988; Barrow \& Götz 1989).

Here we follow the description introduced by Lin, Mestel \& Shu (I965), and used by Icke (1972), for a collapsing Newtonian homogeneous spheroid. Suppose that a particle of such a mass distribution were initially located at $(a, b, c)$, and that at some later time $t$ it had moved to the point $(a X(t), b Y(t)$, $c Z(t))$, then the density $\rho$ would evolve according to

$$
\rho(t)=\rho_{0} / X Y Z \text {. }
$$

The equations of motion for the scaling functions $X, Y$, and $Z$ are found as follows. The potential $\Phi$ obeys

$$
\begin{aligned}
\Phi & =k\left(\alpha x^{2}+\beta y^{2}+\gamma z^{2}\right) \\
& =k\left(\alpha a^{2} X^{2}+\beta b^{2} Y^{2}+\gamma c^{2} Z^{2}\right),
\end{aligned}
$$

and Poisson's equation demands that

$$
k(\alpha+\beta+\gamma)=2 \pi G \rho .
$$


The components of the gravitational force are $-\partial \Phi / \partial x=-(\mathrm{I} / x) \partial \Phi / \partial a$ et cycl., so that the equations of motion become

$$
\begin{aligned}
& -\frac{\mathrm{I}}{X} \frac{d^{2} X}{d t^{2}}=2 \pi G \rho \alpha, \\
& -\frac{\mathrm{I}}{Y} \frac{d^{2} Y}{d t^{2}}=2 \pi G \rho \beta, \\
& -\frac{\mathrm{I}}{Z} \frac{d^{2} Z}{d t^{2}}=2 \pi G \rho \gamma,
\end{aligned}
$$

where the functions $\alpha, \beta$, and $\gamma$ are defined as

$$
\begin{gathered}
\alpha=a b c \int_{0}^{\infty} \frac{d s}{\left(a^{2}+s\right) \Delta} \text { et cycl., } \\
\Delta^{2} \equiv\left(a^{2}+s\right)\left(b^{2}+s\right)\left(c^{2}+s\right),
\end{gathered}
$$

(cf. Chandrasekhar 1969, ch. 3). Here $a, b$, and $c$ are identified with the axes of the ellipsoid.

Now comes a crucial observation, first made by Lynden-Bell (1964): without loss of generality, we can order the axes according to $a>b>c$, in which case $\alpha<\beta<\gamma$, so that equations (6)-(8) give

$$
-\frac{\mathrm{I}}{X} \frac{d^{2} X}{d t^{2}}<-\frac{\mathrm{I}}{Y} \frac{d^{2} Y}{d t^{2}}<-\frac{\mathrm{I}}{Z} \frac{d^{2} Z}{d t^{2}} .
$$

Consequently, the axial ratios $a: b: c$ always increase with time, and slight initial asphericities are amplified during the collapse. This secular increase of aspherical perturbations provides an explanation for the pancake-like, and later filamentary, appearance of large-scale structures (cf. Zel'dovich 1970, 1978). Note also that, for the contraction described, the velocities inside the ellipsoid are linear functions of position: the collapse produces a Hubble-type velocity field.

The equations of motion for the homogeneous ellipsoid were solved numerically by Icke (1972, 1973) for the particular case of prolate spheroidal regions embedded in empty and in Einstein-De Sitter universes. White \& Silk (1979) and Barrow \& Silk (I98I) extended this analysis to general ellipsoids and to arbitrary values of $\Omega$, the density of the background. White $\&$ Silk found an approximate analytic solution of the equations of motion. They clearly illustrated the relationship of the homogeneous ellipsoids to the pancake formalism by showing the close resemblance of the approximate solutions to the linearized equations of Zel'dovich (1970) for the non-linear evolution of a general inhomogeneous density field. A fully analytic treatment was given by Barrow \& Silk (198I), who derived simple analytic expressions for the density contrast at turn-around, the epoch at which pancakes first form, and the infall velocity anisotropy at that epoch.

The homogeneous ellipsoid models neglect the effects of inhomogeneities within the systems considered, as well as the possible role of tidal forces due 
to other nearby objects. White \& Silk (1979) argue that the neglect of the tidal forces is not critical. On the other hand, the quadratic potential approximation will break down as soon as the inhomogeneities on smaller scales become too large. Exactly how and when this occurs depends on the spectrum of the fluctuations. An additional important problem is whether one needs dissipation to keep the pancakes from re-expanding. In the 'pancake picture' of Zel'dovich and collaborators, gaseous dissipation fixates the pancakes, automatically leading to a cellular galaxy distribution, with voids surrounded by walls of galaxies. However, both Dekel (I983) and Peebles (1982) found that even without dissipation pancakes can remain thin.

In order to avoid these complications with the high-density regions, we may view the development of structure in a self-gravitating pressure-free medium by considering the evolution of the low-density regions. These are the progenitors of the observed voids. The arguments presented above can still be applied, except that the sense of the final effect is reverse: because a void is effectively a region of negative density in a uniform background, the voids expand as the overdense regions collapse, while slight asphericities decrease as the voids become larger ('Bubble Theorem', Icke I984). The proof holds strictly only on a non-expanding background, though this should be no objection for structures which are much smaller than the particle horizon. Because $|\delta \rho / \rho|$ does not exceed unity in a void, the approximation will remain good for a longer period except, of course, near the outer parts of the voids, where the matter gets swept up.

The tendency of underdense regions to become more and more spherical in the course of time was first seen in the N-body simulations by Centrella \& Melott (1983) of a three-dimensional neutrino-dominated universe, as well as in numerical calculations of void evolution (Fujimoto I983; Bertschinger 1985).

While the 'Bubble Theorem' provides a useful insight into the qualitative behaviour of a void, it is necessary to carry out more detailed studies in order to get a better understanding of physical quantities like the size distribution of voids in different clustering scenarios (see e.g. Ostriker \& Strassler I989; Zeng \& White I990) and density and velocity profiles in and around voids as a function of time and initial conditions.

Several authors have carried out numerical and analytical studies of the evolution of voids, most of them concentrating on isolated spherically symmetric holes in a uniform background, either with or without compensating ridges. Hoffman \& Shaham (1982) were amongst the first to point out that voids could be a natural outcome of a dissipationless clustering scenario as the result of the evolution of high negative-density peaks in the primordial density field. Peebles (1982), Hoffman, Salpeter \& Wasserman (I983) and Hausman, Olson \& Roth (I983) performed numerical studies of the growth of holes in dissipationless matter. Qualitatively their studies are similar, differing mainly in the density and velocity profile of the matter in and around the void. Peebles (1982) and Hoffman, Salpeter \& Wasserman (1983) took initial density distributions that explicitly include both an underdense core and a compensating overdense region around the core. They showed that when $\Omega \approx \mathrm{I}$ a deep hole can form (Hoffman et al. estimate that $\Omega \geqslant 0.6$ is needed), always surrounded by a dense, thin 
spherical 'ridge'. Alternatively, Hausman, Olson \& Roth (1983) started with an initial density profile without a compensating ridge. They found that even then narrow dense shells will develop in nearly all cases, except if the central perturbation is not strong enough, the initial density profile is not steep enough, or the initial density parameter $\Omega_{i}$ is not high enough. That conclusion was partly challenged by Bertschinger (I985), who argued that dense shells will always form, for both purely compensated or uncompensated holes, if $\Omega=\mathrm{I}$. Fujimoto (I983) performed a similar numerical study. He integrated the equations of motion of a rotating underdense ellipsoid in an expanding background, and found that the underdense region tends to become spherical during its expansion relative to the background, thus illustrating the 'Bubble Theorem'. Ikeuchi \& Umemura (I984) pursued the evolution of negative spherical density perturbations of neutrinos and baryons from decoupling up to the present. The negative-density perturbation of the neutrinos grows quickly to a void and surrounding ridge, whereby shell-crossing occurs. The baryons are forced by gravity to concentrate in the ridges, in which the temperature rises quickly due to dissipation of the kinetic energy.

Analytically, the void evolution was studied by Occhionero, Santangelo \& Vittorio (1983). They used the Tolman solution to describe the evolution of a spherically symmetric hole in an Einstein-De Sitter universe, and found that it agreed quite well with the numerical calculations of Peebles (I982). Extensive analytical calculations of void evolution were carried out by Sato (I982), Maeda, Sasaki \& Sato (I982), Maeda \& Sato (I983), and Sato \& Maeda (1983) within the context of general relativity, using the 'thin wall' approximation. They found asymptotic expansion laws of voids in an Einstein-De Sitter universe of $R \propto t^{0 \cdot 8}$ for adiabatic perturbations, and $R \propto t^{0.794}$ for isothermal perturbations. Suto, Sato \& Sato (1984) extended this work numerically by including pressure. Pressure prevented shell crossing as well as growth of a void below a scale of $10^{-5}$ times the particle horizon scale at recombination. Lake \& Pim (1985) and Pim \& Lake (1986, 1988) generalized the work of Sato and collaborators on the general relativistic thin-wall approximation to non-flat Robertson-Walker backgrounds. They studied the evolution of both vacuum- and radiation-filled spherical voids, both analytically and numerically.

A very extensive study of the evolution of voids in Einstein-De Sitter universes was carried out by Bertschinger (1983, I985). He found similarity solutions for the non-linear evolution of isolated, spherical holes. These grow from initially small negative-density perturbations to a self-similar form after becoming non-linear. Bertschinger found similarity solutions for voids in collisionless (e.g. massive neutrinos or cold dark matter), collisional (e.g. baryons), and mixed gases, for both compensated holes (no net mass deficit at large distances) and uncompensated ones. In all these solutions, shellcrossing and caustics occur in the collisionless component, while shocks form in the collisional fluid. The similarity solution for a compensated hole in collisional gas turns out to be the same solution as for blast waves (Schwarz, Ostriker \& Yahil 1975; Ikeuchi, Tomisaka \& Ostriker I983; Bertschinger I983; see also Ostriker \& Cowie I98 I ; Ikeuchi I98 I ; for a review see Ostriker \& McKee 1988). Independently, Fillmore \& Goldreich (I984) derived 
similarity solutions for spherically symmetric voids in an Einstein-De Sitter universe filled with cold, collisionless matter by numerical integration of the equations of motion. They reproduced the similarity solution found by Bertschinger, and in addition found that the character of the solution depends upon the profile of the initial density deficit: gradual perturbations give rise to holes within which the density rises smoothly to the background value, while steep perturbations result in voids bounded by overdense shells with sharp edges. Bertschinger (1985) also discussed several processes which might modify the obtained similarity solutions. Evaluating the effects of nonspherical symmetry, finite velocity dispersion, cooling and $\Omega \neq I$ cosmological backgrounds by numerical simulations and heuristic arguments, Bertschinger argued that they do not much affect the expansion of voids. The exception is the case $\Omega \ll \mathrm{I}$, in which the expansion in comoving coordinates will stop. Numerical simulations with a two-dimensional axisymmetric hydrodynamics code showed that non-spherical holes tend to become more spherical as they evolve, which confirms the 'Bubble Theorem'.

Just as in the case of growing filaments, the velocity field inside the voids (not in ridges surrounding them), in the regime where the quadratic potential approximation is valid, is proportional to the distance inside them. The numerical simulations by Peebles (1982) and Hoffman, Salpeter \& Wasserman (I983) confirm this. Thus, voids can be described as 'superhubble bubbles'. This has non-trivial observational consequences, because the linearity makes it impossible to separate local void expansion from true cosmic Hubble motion. Hence, considerable deviations from the mean Hubble flow may go undetected until the observations encompass a scale larger than that of the voids. When such scales are reached, the void expansion will appear in the form of large-scale streaming motions. The corresponding velocities are evidently on the order of

$$
v \approx \varepsilon D H_{0},
$$

where $D$ is the size of the void and $\varepsilon$ is the local excess of the expansion over the mean Hubble motion. The value of $\varepsilon$ is not known in detail, but might be about $0.1-0.2$ (Centrella \& Melott 1983 ; Bertschinger 1985). If $D$ is about $50 \mathrm{Mpc}$ (De Lapparent, Geller \& Huchra I986), we find that $v \approx 500 \mathrm{~km} \mathrm{sec}^{-1}$, taking $H_{0}=75 \mathrm{~km} \mathrm{sec}^{-1} \mathrm{Mpc}^{-1}$. This number does, however, depend on assumptions about the dependence of the mass-to-light ratio on the local density ('biasing').

According to the above, taking voids as the dominant dynamical component of the Universe, we may think of the large-scale structure as a close packing of spheres of different sizes, out of which matter flows in a slightly super-Hubble expansion towards the interstices of the spheres. Thus, the importance of the Bubble Theorem is that it provides a specific physical mechanism for producing the non-Poissonian matter distribution in the largescale Universe.

\section{VORONOI FOAM}

We have now found at least a partial answer to the question: If the distribution of galaxies is not Poissonian, then what is it? Continuing the 
above argument, we can construct the 'skeleton' of the mass distribution by considering the locus of points towards which the matter streams out of the voids. Suppose that some cosmic process produces a collection of regions where the density is slightly less than average (the origin and statistical properties of the requisite fluctuations is a very important unsolved problem). As we have seen, these regions are the seeds of the voids, because underdense patches become expansion centres, from which matter flows away until it encounters similar material flowing out of an adjacent void. Making the approximation that the excess Hubble parameter is the same in all voids, the matter must collect on planes that perpendicularly bisect the axes connecting the expansion centres.

For any given set of expansion centres, or nuclei, the arrangement of these planes defines a unique process for the partitioning of space, a Vorono tessellation (Voronoi I908). A particular realization of this process (i.e. a specific subdivision of $N$-space according to the Voronoi tessellation) may be called a Voronoi foam (Icke \& Van de Weygaert 1987). In three dimensions a Voronoi foam consists of a packing of Voronoi cells, each cell being a convex polyhedron enclosed by the bisecting planes between the nuclei and their neighbours. A Voronoi foam consists of four geometrically distinct elements: the polyhedral cells (voids), their walls (pancakes), edges (filaments) where three walls intersect, and nodes (clusters) where four filaments come together.

This mathematical construct is used in many other fields, e.g. molecular physics, metallurgy, geology and forestry (cf. Meyering I953; Miles 1970; Stoyan, Kendall \& Mecke 1987). Due to these diverse applications it has acquired a set of alternative names, such as Dirichlet regions (Dirichlet I850), Wigner-Seitz cells, and Thiessen figures. Kiang (I966) was the first to apply Voronoi tessellations to astrophysics, in his study of the mass spectrum obtained in the fragmentation of interstellar clouds. Matsuda \& Shima (I984) were the first to propose the use of Voronoi tessellations in cosmology. They pointed out the similarity between two-dimensional Voronoi tessellations and the outcome of numerical clustering simulations, in particular of the simulation of a neutrino-dominated universe by Melott (I983). However, they did not explicitly supply the physical mechanism behind this phenomenon.

In the cosmological context each Voronoi cell is considered to be a void. The planes are identified with the 'walls' in the galaxy distribution (see, e.g. Geller \& Huchra 1989), the filaments are identified with the elongated 'super' clusters (Icke 1972; Oort 1983; Giovanelli, Haynes \& Chincarini I986), and the vertices correspond to the virialized Abell clusters.

We have constructed two- and three-dimensional Voronoi foams geometrically (Icke \& Van de Weygaert 1987; Van de Weygaert \& Icke I989). The example in Fig. 3 shows the characteristic appearance of twodimensional Voronoi foams; the similarity with 2-D numerical simulations of gravitational clustering of collisionless particles is indeed quite striking (Melott 1983; Matsuda \& Shima 1984). In three dimensions, the similarity is even more remarkable, in projection as well as in a slice (compare Figs. 2 and 6). A stereogram of three geometrically constructed Voronoi cells is given in Fig. 4, showing the disposition of the walls, filaments and nodes. 

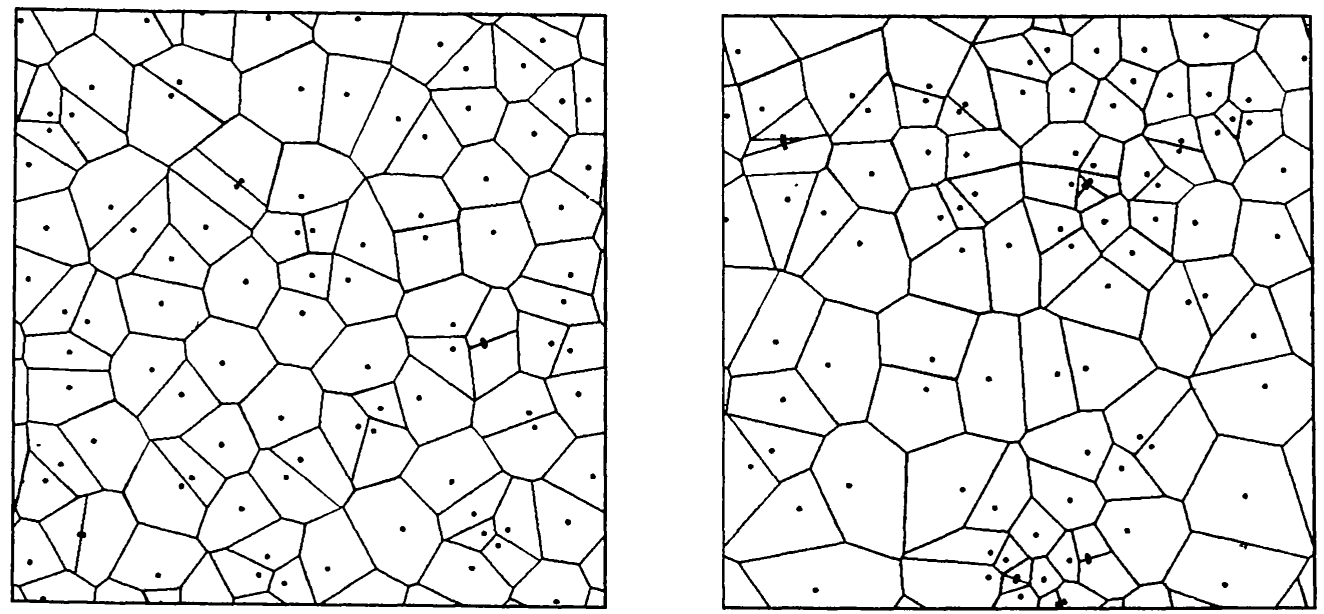

FIG. 3. Two examples of two-dimensional Voronoi foams, for different values of the correlation between the nuclei, which are indicated by dots. After Icke \& Van de Weygaert (1987).
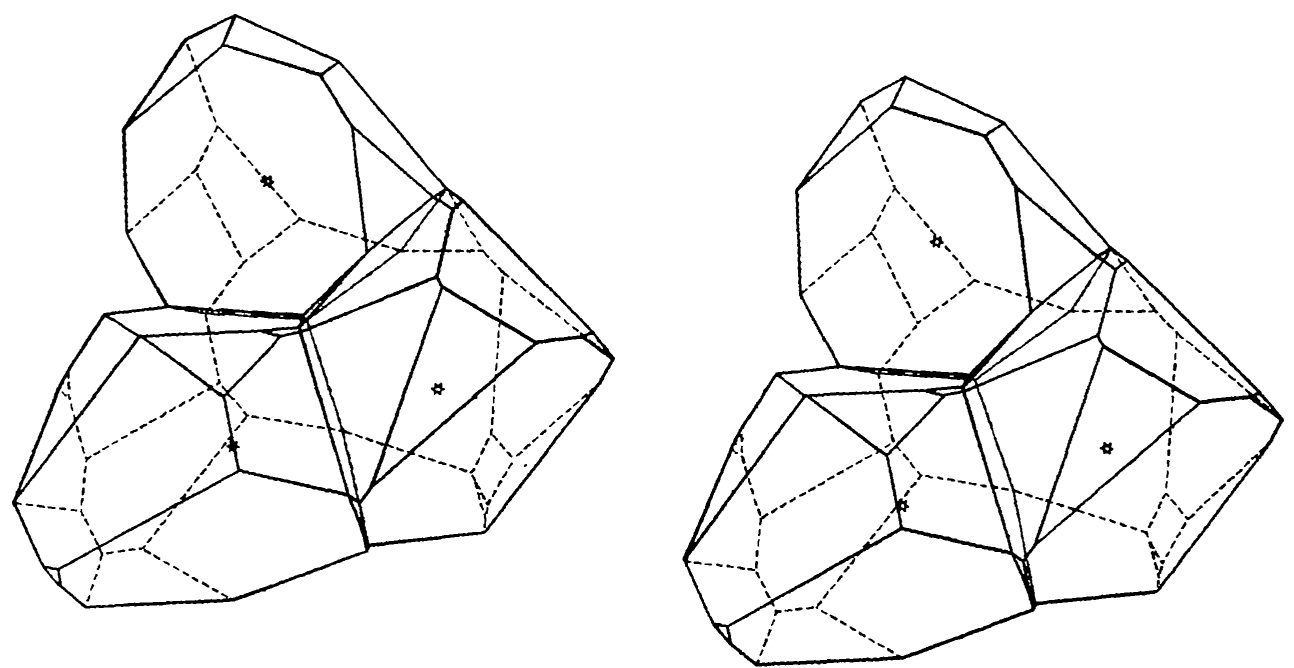

Fig. 4. Stereo pair of three Voronoi cells and their nuclei (stars). The cell edges ('filaments') are drawn in thick lines, the edges of the three walls where the cells touch are thinly drawn, and hidden lines are dashed.

The advantage of using these geometrically constructed models is that one is not restricted by the resolution or number of particles. A cellular structure can be generated over a part of space beyond the reach of any $\mathrm{N}$-body experiment. This makes the Voronoi model particularly suited for studying the properties of galaxy clustering in cellular structures on very large scales, for example in very deep pencil-beam surveys, and for studying the clustering of clusters in these models.

If one relaxes the constraint of equal excess Hubble expansion in every void, one obtains a Johnson-Mehl tessellation (Johnson \& Mehl I939), which would be more realistic. This structure closely resembles the cells produced in the 'adhesion' model by Kofman \& Shandarin (1988). In Johnson-Mehl tessellations the walls are not bisecting planes but hyperboloids with their 
axes along the lines connecting the nuclei. It is very difficult to construct such a tessellation geometrically, but one might do it approximately by using a kinematical approach* such as the one described in the next section. It will be interesting to see how large the differences of the statistical properties of Johnson-Mehl and Voronoi tessellations are. But it is not a priori clear whether Johnson-Mehl tessellations are a real improvement, or whether they just complicate the calculations, while losing the attractive simplicity of the Voronoi model.

The Voronoi foams are expected to give a good asymptotic description of the matter distribution at late times in several physical models. Models to which the Voronoi foams apply can be either gravitational instability models in which the structure formation is dominated by the negative-density fluctuations, as in models with fluctuation spectra having a high-frequency cutoff or considerable power at low wavelengths; or models in which the driving force is due to explosions. The Voronoi foams outline the 'skeleton' of the mass distribution, around which matter assembles during the evolution of the Universe.

\section{A KINEMATICAL MODEL OF VORONOI CELL FORMATION}

As argued in the previous section, the Voronoi tessellation can be considered as the skeleton of the mass distribution. The galaxy distribution itself depends on the initial fluctuation spectrum, and on the details of the small-scale interactions of the gravitating matter. N-body simulations are preferred for solving that problem, but even those may not suffice. It seems probable that the small-scale structure in the baryonic matter is considerably influenced (maybe even determined) by dissipational processes. But it can be very useful to distribute galaxies on a Voronoi skeleton according to some plausible description, in order to study the clustering properties in a cellular model of large-scale structure.

In this way a better understanding of the outcome of statistical measures from observational catalogues or N-body simulations can be achieved. For example, one might use a uniform distribution within each wall, as was done by Yoshioka \& Ikeuchi (I989), Weinberg (I989), and Van de Weygaert (I99I). One may also resort to a more realistic distribution based on an idealized description of dynamical simulations. Such a model is the kinematical model used by Van de Weygaert \& Icke (I989).

The kinematical model is based on the notion that when matter streams out of the voids towards the Voronoi skeleton (i.e. the mathematical tessellation corresponding to a given distribution of expansion centres), cell walls form when material from one void encounters that from an adjacent one. If the matter is collisionless (as in some species of dark matter), only selfgravity will tend to hold it together. Moreover, if galaxies form during the expansion of the voids, the formation of the walls may be amplified by

* This was done by Icke (unpublished, I989); it is found that the difference is indeed small, due to the fact that the deviation between a hyperboloidal and a plane wall is of second order in the 'excess Hubble constant' of the voids. 


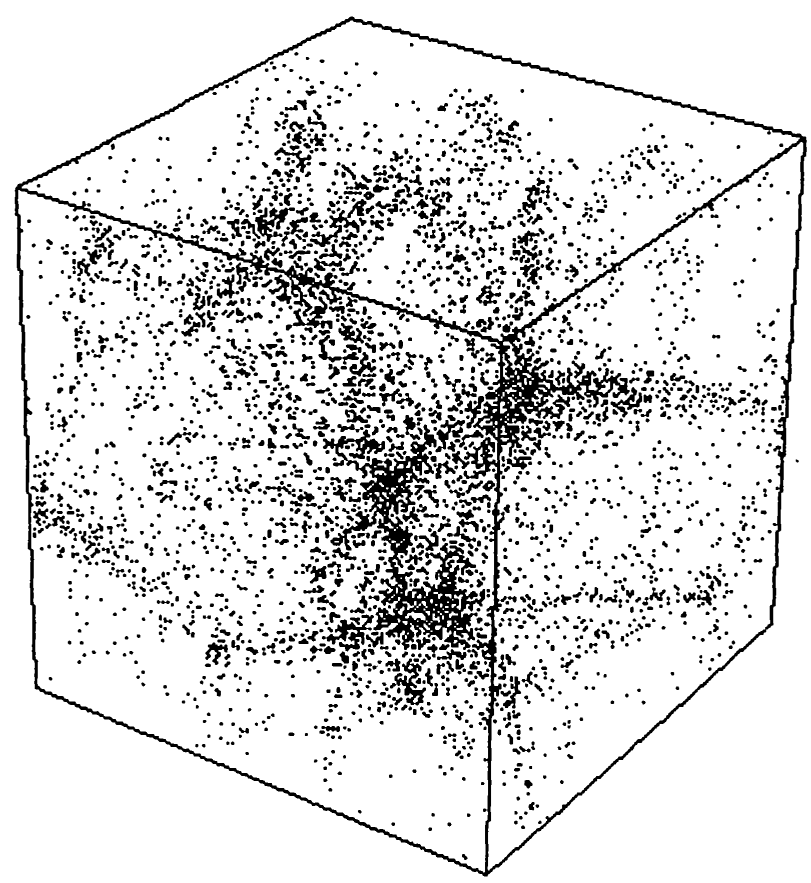

Fig. 5. Perspective cube showing the distribution of 10000 galaxies expanding away from I5 Voronoi nuclei, at dimensionless time $t=2$; the amplitude of the galaxy-galaxy two-point correlation function at this time corresponds approximately to what is observed today.

dynamical friction between the galaxies (Toomre \& Toomre 1972; Binney \& Tremaine 1987, ch. 7).

Accordingly, the structure formation scenario of the kinematical model is as follows. Within a void, the mean distance between galaxies increases uniformly in the course of time; this amounts to an excess Hubble expansion about the cell nucleus. When a galaxy tries to enter an adjacent cell, the gravity of the wall, aided and abetted by dissipational processes, will slow its motion down. On the average, this amounts to the disappearance of its velocity component perpendicular to the cell wall. Thereafter, the galaxy continues to move within the wall, until it tries to enter the next cell; it then loses its velocity component towards that cell, so that the galaxy continues along a filament. Finally, it comes to rest in a node, as soon as it tries to enter a fourth neighbouring void. In a Voronoi foam, there are exactly four cells adjoining each node, and the above process is unique. An immediate consequence of this kinematic behaviour is that the density in the walls quickly becomes smaller than in the filaments which, in turn, remain less dense than the nodes, where all matter eventually congregates. This is the main reason why we identify the nodes with the rich Abell clusters.

Voronoi cell formation with periodic boundary conditions, as described above, is shown in Fig. 5. When taking slices, as in Fig. 6, one sees a striking resemblance with the observed redshift distributions of galaxies (e.g. De Lapparent, Geller \& Huchra 1986; see Fig. 2, reproduced from Geller \& Huchra 1989). Of course, the picture is expected to differ considerably in the non-linear areas. In particular, the velocity fields near the clusters and 


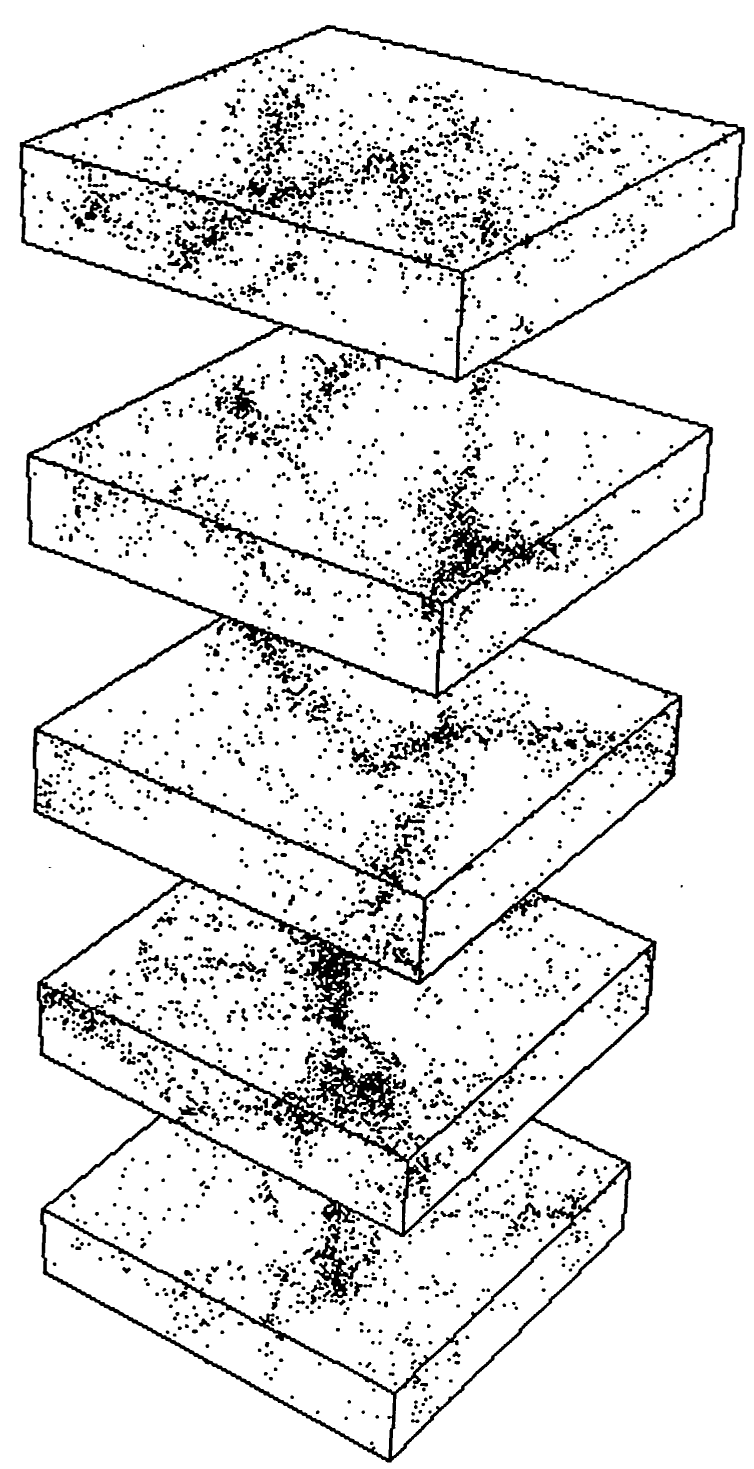

FIG. 6. The cube of Fig. 5, sliced into five equal slabs.

filaments are expected to be dominated by their gravity, and not by the expansion of the void.

\section{COMPARISON WITH OBSERVATIONS: CLUSTERING STATISTICS}

To quantify the comparison between the Voronoi tessellation and observations, one has to derive the statistical properties of the clustering. Quantification of the complicated three-dimensional pattern in the largescale galaxy distribution, however, has proved to be a difficult task. A complete statistical description of any point process demands higher order $\mathrm{N}$-point correlation functions (Peebles 1980 ), yet it is practically impossible to measure $N \geqslant 4$ correlation functions from galaxy catalogues. This has led to the use of several techniques highlighting different geometrical properties.

The most widely used statistic is the two-point correlation function, $\xi(r)$, 
and its sky-projected equivalent, the angular two-point correlation function $w(\theta)$. The two-point correlation function provides a straightforward measure of deviations from Poissonian statistics. It is defined such that the number $\delta N$ of objects found in a spherical shell with volume $\delta V$ and radius $r$, centred on a randomly chosen object, is

$$
\delta N=n[\mathrm{I}+\xi(r)] \delta V,
$$

where $r$ is the distance between the two volume elements and $n$ is the average number density of objects. The popularity of $\xi(r)$ can be understood from its direct relationship to the dynamics of the matter distribution on small scales, for $\xi \gg$ I, via the cosmic virial theorem, and the hope that on large scales, where the clustering is weak $(\xi \ll I), \xi$ can be related directly to the perturbation power spectrum of Gaussian fluctuations.

In the case of galaxies, the value of the mean density $n$ is unknown, in part because the Universe has been insufficiently sampled (the deeper the redshift surveys go, the larger structures they reveal: cf. Geller \& Huchra 1989). For the CfA I survey Davis \& Peebles (1983) found

$$
\begin{gathered}
\xi_{g g}(r)=\left(r_{0} / r\right)^{\gamma}, \\
\gamma=\mathrm{I} \cdot 77 \pm 0.04 ; \quad r_{0}=(5.4 \pm 0.3) h^{-1} \mathrm{Mpc},
\end{gathered}
$$

so that it seems fairly certain that $\xi$ can be adequately described by a power law over a decade and a half in $r$. In particular, there is no good evidence for the existence of a preferred length-scale in the small-scale galaxy clustering, though there are some indications from the CfA2 survey that $\xi_{g g}$ has a shoulder at a few megaparsec.

The form of $\xi_{g g}$ is most convincingly shown by the power-law behaviour of the angular correlation function $w(\theta)$. We can learn a lot from $w(\theta)$, because it can be determined from far larger samples of galaxies, and because it is intimately coupled to $\xi_{g g}$ by Limber's equation (Limber 1954). Both Groth \& Peebles (1977), in the case of the Lick survey, and Maddox et al. (I990) in the APM survey, find a power law behaviour of $w(\theta)$ up to around $3^{\circ}$, and a break afterwards (although they disagree on the sharpness of the break).

This is quite surprising, because just looking at the redshift surveys shows that there is a lot of structure on scales of at least $50 \mathrm{Mpc}$. Filamentary structures have been studied using percolation analysis (Zel'dovich, Einasto \& Shandarin 1982), and more recently using the cleaner method of Minimal Spanning Trees (Barrow, Bhavsar \& Sonoda 1985; Ling 1987; Bhavsar \& Ling 1988). Although looking quite promising at first, studies of the percolation method by Bhavsar \& Barrow (1983) and Dekel \& West (1985) showed that percolation statistics suffer from severe problems. Percolation does not distinguish well between some patterns which appear clearly different to the eye, while they are dependent on sampling parameters (mean number density and sample depth) in a way which is unknown a priori because the test depends on the same properties which it ought to measure. Minimal Spanning Trees seem to do a better job in detecting filamentary structures, while their recently discovered connection to the dimensional properties of the sample (Martínez et al. I990; Van de Weygaert, Jones \& 
Martínez I99I) may lead to a better understanding of their connections to other statistical measures.

Gott and collaborators studied the topology of the galaxy distribution by characterizing it by the mean Gaussian curvature per unit volume of surfaces of constant density. This method was introduced by Gott, Melott \& Dickinson (1986), and discussed at length by Melott, Weinberg \& Gott (1988). Based on a small volume-limited sample of galaxies, Gott et al. reached the conclusion that the topology is sponge-like, meaning that the high- and low-density regions are both connected, as opposed to a purely cellular topology, in which only the high-density regions are connected. Although this descriptor looks very promising, its application suffers from small-number statistics (and from insensitivity to underlying statistics: Coles \& Jones I99I).

A potentially very powerful method is the void probability function (VPF), and the related number count distributions, as different models predict large differences. White (1979) showed that the VPF, the probability that a randomly placed volume $V$ is empty, depends on correlation functions of all orders. The VPF has been determined for angular and redshift catalogues of galaxies by several groups (e.g. Bouchet \& Lachièze-Rey I986; Maurogordato \& Lachièze-Rey 1987). The VPF is interesting because it is a combination of the correlation function and the pattern recognition approach to clustering statistics. A disadvantage is that one needs quite large samples to estimate the VPF with confidence, in particular at low values, so that its use is as yet rather limited.

Another promising method is the description of galaxy clustering in terms of its generalized, fractal dimensions (Jones et al. I988; Martínez \& Jones I990; Martínez et al. 1990). By using the minimal spanning tree as an estimator (Van de Weygaert, Jones \& Martínez I99I), Martínez \& Jones (I990) showed that the Hausdorff dimension $D_{H}$ of the galaxy distribution, which one may consider as the 'geometrical' dimension, in the CfAI catalogue is $D_{H} \simeq 2 \cdot I \pm 0 \cdot I$, differing significantly from its correlation dimension $D_{2}\left(D_{2}=3-\gamma \simeq \mathrm{I} \cdot 3 \pm 0 \cdot \mathrm{I}\right)$. This means that the spatial distribution of galaxies cannot be described by a simple fractal as advocated by Coleman, Pietronero \& Sanders (I988), but possesses a more complex, possibly multifractal, structure. A nice feature of the multifractal method is that theoretical models can be compared quantitatively with observations on the basis of at least two numbers, $D_{H}$ and $D_{2}$, instead of just the two-point correlation function, providing a far better discriminating power (see Martínez et al. 1990).

Very interesting and useful could be the use of the Voronoi tessellation itself, and its dual, the Delaunay tessellation, as a tool in studying the higherorder clustering properties of a point process. The statistics of the geometrical properties of two-dimensional Voronoi tessellations were studied in Icke \& Van de Weygaert (1987). One of the conclusions was that the distribution of angles between edges is very sensitive to the underlying point process. In fact, Ling (1987) used this approach in his thesis and illustrated its use in two dimensions on artificial point processes, the CfA catalogue, and a CDM simulation. He concluded that the statistic is very flexible, allowing a scaledependent study of the distribution. However, the translation of this 
statistical information to more familiar statistical descriptors is not straightforward, and might prohibit an extensive use of the Voronoi distribution as a tool in statistics.

Evidence for structure on large scales is also provided by the two-point correlation function of Abell clusters, which has the same slope as, but a far larger amplitude than, the galaxy-galaxy correlation (Bahcall \& Soneira I983; Klypin \& Kopylov I983; Postman, Geller \& Huchra I986):

$$
\begin{gathered}
\xi_{c c}(r)=\left(r_{0} / r\right)^{\gamma}, \\
\gamma=\mathrm{I} \cdot 8 \pm 0 \cdot 2 ; \quad r_{0}=(26 \pm 4) h^{-1} \mathrm{Mpc},
\end{gathered}
$$

up to a scale of $\mathrm{I} 0 \mathrm{~h}^{-1} \mathrm{Mpc}$ (taken from Bahcall I988). Although there are some indications that $r_{0}$ in equation (I7) is an overestimate, due to contamination effects in the Abell catalogue (Sutherland 1988; Dekel et al. 1989; Olivier et al. 1990), the difference in amplitude between $\xi_{c c}$ and $\xi_{g g}$ suggests that at least one of them does not properly sample the underlying mass distribution. The difference in amplitude can, of course, be attributed to a dependence of the mass-to-luminosity ratio on the local mass density ('biasing'; see, e.g. Kaiser I984).

We now proceed to show that the cluster-cluster correlation function could also be due to the cellular geometry of a Voronoi distribution.

\section{CONFRONTING THE VORONOI MODEL WITH OBSERVATIONS}

Comparing observations of the galaxy distribution and the theory of the evolution of the gravitational field requires knowledge of how light traces the gravitational potential; in other words, knowledge of the biasing. As we do not know what the dark matter is, nor how its kinetic behaviour differs from that of the baryons, we assume for simplicity that light traces mass faithfully. However, our main result (the pair correlation behaviour of Abell clusters and of Voronoi vertices) does not depend on this, provided that the dark mass and the luminous matter are positively correlated.

In order to compare the Voronoi model with the statistical properties of the observed galaxy distribution, we have determined the two-point correlation functions of the kinematic simulations and of the Voronoi vertices (Van de Weygaert \& Icke I989).

Because we identify the vertices with Abell clusters, the comparison could be done without further assumptions. When doing this, we can use the fact that the Voronoi node distribution is a topological invariant in co-moving coordinates, and does not depend on the way in which the walls, filaments, and nodes are populated with galaxies. Thus, the statistics of the nodes should provide a robust measure of the Voronoi properties.

For a random distribution of 1000 expansion centres, generating a Voronoi tessellation with 6733 vertices, we got the remarkable result shown in Fig. 7. In order to make a proper comparison between theory and observations, we must specify a single scaling parameter, with the dimension of length: the only free parameter of the Voronoi tessellation can always be specified in this way. We might, for example, choose the mean distance between nuclei. Observationally, however, this would be a very awkward choice. Instead, we applied our scaling by expressing $r$ in units of $d / 2$, where 

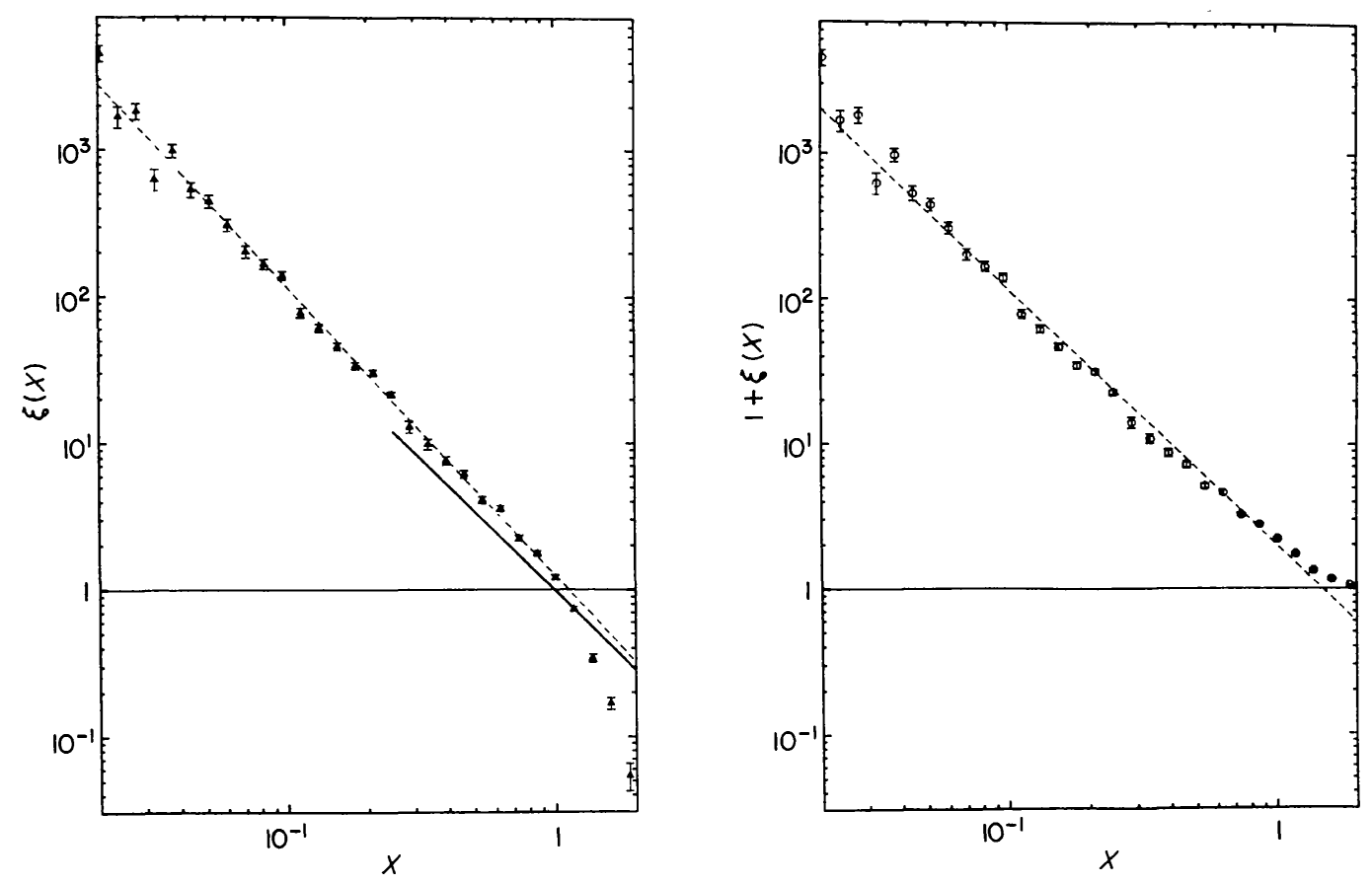

FIG. 7. The spatial vertex-vertex correlation function of a Voronoi foam with 1000 Poissonian nuclei. The left panel shows $\xi$, the right one $\mathrm{I}+\xi$. Both have been plotted as functions of the dimensionless quantity $x \equiv 2 r / d$, in which $d$ is the mean separation between vertices. The heavy solid line is an approximate fit to the observational data.

$d$ is the mean separation between vertices (in the model) or clusters (in the observations). The vertex-vertex correlation function we obtained is a power law from small scales up to the scale of one Voronoi cell, beyond which it vanishes:

$$
\begin{gathered}
\xi_{v v}(r)=\left(r_{0} / r\right)^{\gamma}, \\
\gamma=2 \cdot 0 ; \quad r_{0}=\mathrm{I} \cdot \mathrm{I} 3
\end{gathered}
$$

(in dimensionless units; taking $d$ equal to $55 h^{-1} \mathrm{Mpc}$ for Abell clusters of richness class $\mathrm{R} \geqslant \mathrm{I}$ we get $r_{0}=3 \mathrm{I} h^{-1} \mathrm{Mpc}$ in physical units). The uncertainties shown in Fig. 7 are purely Monte Carlo scatter; we have not performed other estimates of the uncertainty of the calculations, such as bootstrap resampling, because the Monte Carlo spread is very small already (and is, in any case, very much smaller than the scatter of the correlation function derived from observations).

Thus, both the slope $(\approx-2)$ and the amplitude of the vertex-vertex correlation function are in good accordance with the cluster-cluster correlation of Bahcall \& Soneira. Although several other models of large scale structure formation, including the popular cold dark matter scenario (e.g. White et al. 1987), have succeeded in obtaining the right slope, they did not succeed in reproducing the amplitude. Thus, it is remarkable that we have reproduced the amplitude of the correlation function. It should be noted that this fit is not obtained arbitrarily, but comes about spontaneously by choosing the natural normalization in which the mean density of Voronoi vertices equals the observed density of Abell clusters. 
The normalization, which gives $r_{0}=3 \mathrm{I} h^{-1} \mathrm{Mpc}$, also determines the average size of the Voronoi cells. When this size is used in a calculation of the distribution of redshifts of galaxies populating Voronoi foam in a deep pencil-beam survey, the results obtained are in excellent agreement with the observations (Broadhurst et al. 1990; Coles I990; Van de Weygaert 199I). We will return to this below.

The slope is not very sensitive to the details of the stochastic process which determines the distribution of the nuclei. When we looked at the vertices of Voronoi tessellations based on non-Poissonian, clustered, expansion centres, we found that they also had power-law correlation functions, with slopes and amplitudes which only vary mildly with the correlation properties of the nuclei.

The broad conclusion we draw from this is that the cluster-cluster correlation function is determined by the geometrical properties of the galaxy distribution, i.e. their cell-like or sponge-like arrangement. Because this conclusion is purely based on the geometry of the structures which have formed, instead of their dynamics, we consider this as the key result in the study of the Voronoi tessellations and their relation to large-scale structures.

The Voronoi vertices not only reproduce the cluster-cluster two-point correlation function very well, but their superclustering properties as expressed in the cluster multiplicity function are remarkably similar to the observed ones too. By identifying superclusters in a percolation algorithm, one can find the percentage of clusters lying in superclusters of a certain size. Batuski, Melott \& Burns (1987) had concluded that all 'standard' models (CDM, HDM and isothermal) failed to reproduce the Abell catalogue multiplicity function by large margins. In this respect it is striking that the Voronoi vertex multiplicity function is within 6 per cent of the observed one.

To give an impression of the distribution of our 'Voronoi clusters', we have plotted in Fig. 8 some I 700 Voronoi vertices projected onto the sky, within an area of 4.13 steradian, which should be compared with the projected distribution of 1682 Abell clusters, constituting a complete statistical sample on the Northern Hemisphere (Bahcall I988). Although the eye is not the best statistical tool, the general conclusion is that the Voronoi vertex catalog resembles the Abell catalog quite well, thereby visually confirming the statistical tests.

Another test of the Voronoi model will be the topology of the galaxy distribution. In such analyses one should distinguish between the mathematical Voronoi skeleton and the actual mass density with which that skeleton is covered. Weinberg, Gott \& Melott (1987) used a Voronoi foam with galaxies distributed uniformly within each wall (but with a number density differing from wall to wall, being proportional to the distance of the wall from the nucleus). They showed that this model has a clear non-Gaussian 'bubble' topology, with isolated negative contour surfaces, and multiply connected positive contours. In the kinematical model described in Section 5 the walls become porous, leading to a matter distribution which is much more sponge-like. This will be tested using the same topology algorithm in a future study.

Recently Broadhurst et al. (I990) found a striking, quasi-periodic, galaxy redshift distribution in a pencil-beam survey. The mean spacing between the 


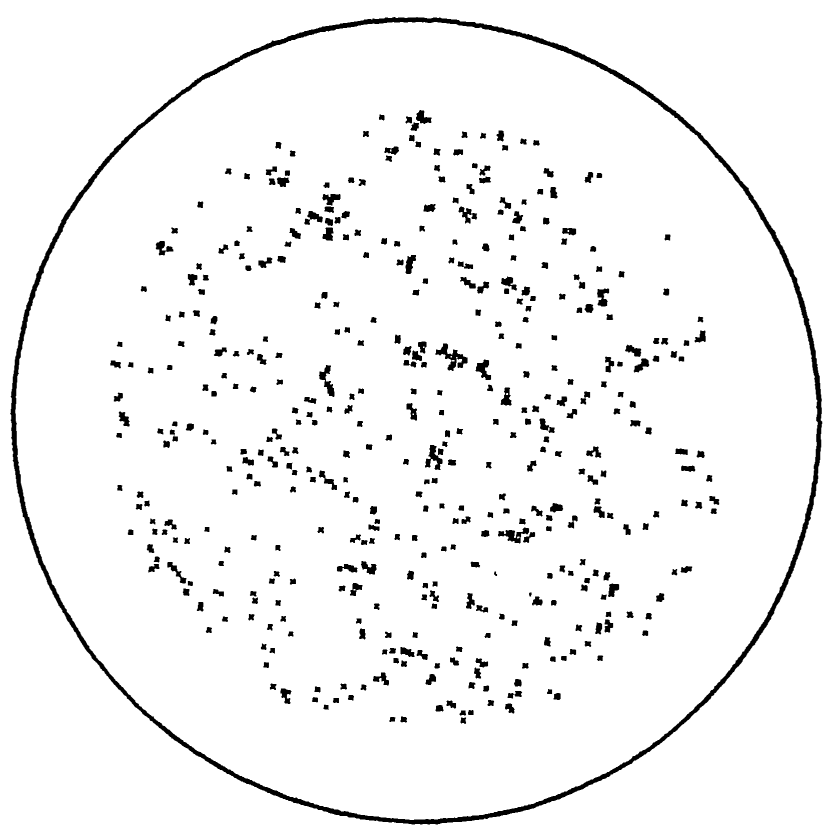

FIG. 8. Projected distribution of 1700 Voronoi vertices on the sky within a solid angle of 4.13 steradian. The projected distribution of I 682 Abell clusters on the Northern Hemisphere within 4.26 steradian, with which this should be compared, can be found in Bahcall (1988).

peaks in the redshift distribution was found to be $128 h^{-1} \mathrm{Mpc}$. Another remarkable discovery was the evidence for 'pleated walls' spanning hundreds of megaparsec (Geller \& Huchra 1989; Fig. 2). Both of these phenomena arise quite naturally within a Voronoi model. In the Voronoi picture the 'Great Wall' occurs because adjacent Voronoi walls form large pleated sheets. Both Coles (1990) and Van de Weygaert (I99I) interpreted the Broadhurst et al. results on the basis of the Voronoi model. Coles (1990) used some new analytical results on Voronoi tessellations derived by Møller (1989). He showed that in a Voronoi foam resulting from Poissonian nuclei with a mean density of $n \simeq\left(100 h^{-1} \mathrm{Mpc}\right)^{-3}$ (i.e. using the normalization we discussed above), the mean distance between intersections of different cells is $\left\langle\lambda_{1}\right\rangle \simeq \mathrm{I} 37 h^{-1} \mathrm{Mpc}$, while he estimated that along about 5 per cent of the lines of sight one can expect a more or less 'periodic' redshift distribution.

These analytical estimates were confirmed by the Monte Carlo simulations of Van de Weygaert (199I). He computed a three-dimensional Voronoi tessellation resulting from 2500 Poissonian nuclei. Galaxies, whose luminosities are selected from a Schechter luminosity function, were placed randomly within the walls of the cellular model, while taking account of the proper density in each topological feature. Simulations of deep magnitudelimited pencil-beam surveys (cones) through these structures were performed. The redshift distribution in two opposite beams, showing periodicity, is shown in Fig. 9, together with the corresponding pair counts. On the basis of some 30 pairs of opposing beams out to a depth of $z=0.5$ it is estimated that 15 per cent of the beams show the observed regular pattern, with a spacing between the peaks on the order of $105^{-1} 5 h^{-1} \mathrm{Mpc}$ (normalizing on the mean distance between clusters, as described above). 

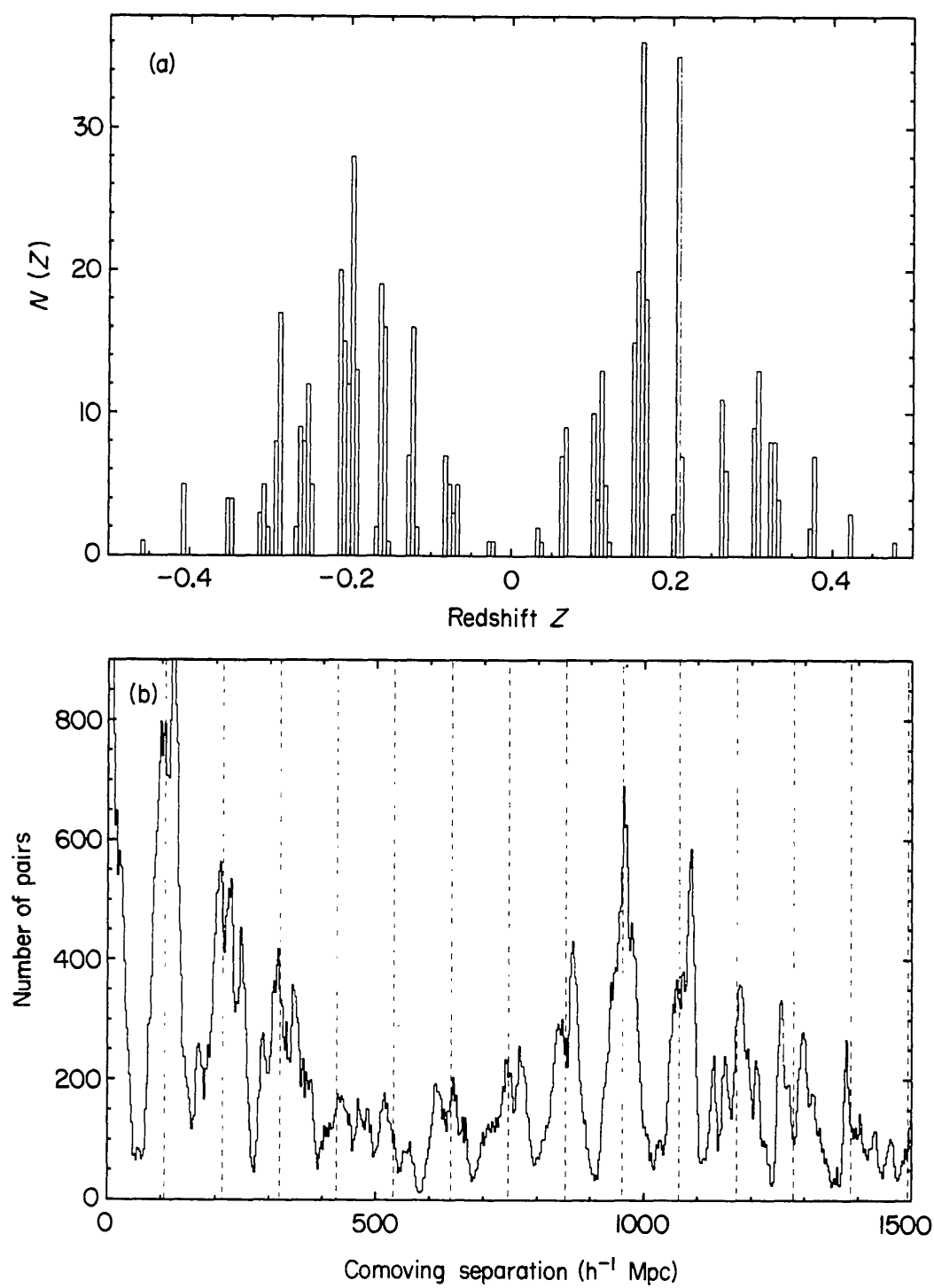

Fig. 9. A deep pencil-beam redshift survey through a Voronoi foam $\left(m_{11 m}=2 \mathrm{I} \cdot 5\right)$. (a) The redshift distribution for pencil beam surveys taken in opposite directions through a 3-D Voronoi tessellation. Positive redshift means the galaxy is in the cone pointing in one direction, while negative redshift means the opposite direction. (b) The corresponding pair-count correlation diagram. The dashed lines indicate scales as multiples of $107 h^{-1} \mathrm{Mpc}$.

Most pencil beams show striking peaks in the galaxy distribution, on the same scale, but without periodicity. It is striking that both the quasiperiodicity, and its observed scale, can be explained easily within the Voronoi picture.

The Voronoi model has so far met several statistical tests (see also the following section), but a grave objection was raised by Coles \& Barrow (1990) concerning the smoothness of the cosmic microwave background radiation (CMBR). They estimated the redshift at which void shells coalesce in a universe filled with homogeneous undecelerated expanding shells around Poissonian nuclei (assuming that the self-similar non-linear expansion phase is not yet reached), whilst requiring that the voids did not percolate at 
recombination; that the voids grow large enough to coalesce (i.e. cellular large-scale structure has formed); and that the temperature fluctuations of the CMBR produced by the primordial underdensities are less than the observed limits.

Coles \& Barrow found that the Sachs-Wolfe effect leads to fluctuations which are only marginally in agreement with observations if a period of reionization is invoked. In that case a redshift of coalescence of $z_{\mathrm{c}} \approx 5.16$ is obtained, while Saarinen, Dekel \& Carr (I987) showed that $z_{\mathrm{c}} \sim 5^{-\mathrm{IO}}$ is needed to give the shells time enough to cool and fragment. Moreover, for adiabatic density fluctuations, or fluctuations with non-zero velocities on the last scattering surface, the model is completely excluded by small-angle limits, unless there is extensive re-ionization. However, such a process should have left its marks on small angular scales by the Sunyaev-Zel'dovich effect and as anisotropies in the X-ray background. It is not clear yet whether these would exceed the observational limits.

We find these objections very serious, even though they might depend on the specific model of void evolution which Coles \& Barrow (I990) analysed. CMBR fluctuations in our kinematical Voronoi model might behave differently, because in that case the walls form by the gradual crossing of particle orbits, instead of the crossing of material shells. A detailed study of the CMBR in an evolving kinematic Voronoi distribution is under way (Icke \& Poelman I99I); preliminary results show CMBR peak-to-peak fluctuations in the range between $\Delta T / T \approx \mathrm{IO}^{-4}$ and $\Delta T / T \approx \mathrm{IO}^{-2}$, depending on when the Voronoi evolution is assumed to begin. Thus, it appears that the objections by Coles \& Barrow will be sustained. The usual recourse to dark matter and biasing would help, but at the expense of simplicity.

Other ways out may be found by using very non-Gaussian primordial fluctuations, or in the assistance of the gravitational expansion by early explosions. The limits are far less stringent for explosion models, in which only the baryonic component needs to expand, accelerated by the explosive energy from an ad hoc population of exploding objects.

Similar conclusions were reached by Hoffman, Salpeter \& Wasserman (I983) for their model of a honeycomb universe resulting from expanding holes centred on initial underdense seeds on a regular lattice (a degenerate Voronoi tessellation). They determined the underdensity at the cell centres at recombination required to reach a density contrast between the void interior and the mean density of the Universe of $\rho_{c} / \bar{\rho} \approx 0 \cdot I$. For $\Omega=I$, the required initial underdensity is $(\delta \rho / \rho)_{\text {rec }} \approx 0.01$, while for smaller $\Omega$ even larger underdensities are required. While they ignore the resulting Sachs-Wolfe effect, the resulting CMBR anisotropies in the case of adiabatic fluctuations are clearly too large. The limits on those fluctuations are decreased substantially by invoking a dominant dark matter component, although it will not solve the problem of the Sachs-Wolfe induced anisotropies. Other possible solutions to the problem include more exotic possibilities, such as a cosmological constant $\Lambda>0$ which is sufficiently large that a "coasting phase' has already occurred, or explosion-driven bubbles instead of gravitationally driven ones.

If none of these alternatives provides a satisfying solution, the interpretation of large scale structure formation dominated by the expansion 
of voids is wrong. This would not only exclude the Voronoi model, but also similar models in which the voids play an important role (like pancake models, or neutrino-dominated models).

\section{RELATION TO OTHER WORK}

The Voronoi model is based on the formation of irregularities in the mass distribution in the early universe, which are amplified by the gravitational force. The statistical properties of a Voronoi distribution can be computed with confidence and ease. We have found that Voronoi distributions do not only look strikingly like the observed galaxy distribution, but also pass the statistical tests.

Our model is a useful description of the outcome of the evolution of a perturbation field with a high-frequency cutoff or with much power on large scales, as in a universe dominated by massive neutrinos (cf. Centrella \& Melott 1983; Centrella et al. 1988). In this sense, it is closely related to pancake theories of galaxy formation, as studied by Zel'dovich (I970), Klypin \& Shandarin (1983), and Buchert (I989). Moreover, the 'adhesion' approach to clustering evolution, based on the use of Burgers's equation, produces a cellular structure of dark matter which bears some resemblance to our Voronoi tessellations (Kofman \& Shandarin 1988; Gurbatov, Saichev \& Shandarin I989; Kofman I989; Kofman, Pogosyan \& Shandarin I990; Weinberg \& Gunn I990; for a review of these models see Shandarin \& Zel'dovich I989). Voronoi models may be very useful in this context because one can easily derive statistical properties with high statistical significance, which is difficult in models involving large computational effort.

Another approach to clustering was taken by Ikeuchi (I98I) and by Ostriker \& Cowie (I98I), who proposed that the bubble-like distribution of galaxies was caused by enormous explosions in the early Universe, driving the matter out of voids, sweeping primordial gas into dense, expanding shells that cool and fragment into galaxies. Taking this view and basing themselves on N-body simulations by Saarinen, Dekel \& Carr (I987), Weinberg, Ostriker \& Dekel (1989) studied the clustering in a universe of interacting shells. In their model the clusters appear at the vertices where three shells intersect; the shells are distributed randomly throughout the volume. By investigating the model as a function of three free parameters, they try to obtain a fit to the observations. Interestingly, they find that the vertex-vertex correlation function is a power law. However, the most successful choice of parameters yields the right slope but an amplitude which is too high. With these same parameters they also succeed in getting a supercluster multiplicity function roughly consistent with the data. A similar model was proposed by Bahcall, Henriksen \& Smith (1989) on the basis of observational considerations.

Yoshioka \& Ikeuchi (1989) and Weinberg (1989) adopted the Voronoi foam as a 'toy model' of large-scale structure in the explosion scenario. The former pursued the explosion scenario by using 64000 particles in a box, which expanded away from a set of 100 expansion centres. The particles were stopped short in either the walls, the filaments, or the nodes of the corresponding Voronoi tessellation. They used the galaxy paths merely as 
convenient markers for the location of the Voronoi features, and did not allow the galaxies to stream along the walls and the filaments, as we did in our simulations. Their goal was to see what the clustering properties of the particles were if they were all situated in either the walls, the filaments, or the nodes. This version of the explosion scenario did produce power laws for the correlation functions, although the slopes were too shallow when all the particles were situated within the walls or the filaments. The vertex-vertex correlation function confirms the slope of -2 which we found, but because their method is not mathematically exact, they retrieve only a limited subset of vertices (only around 670 in the case of roo voids). Thus, they could not study the correlation function over a large range, or estimate the amplitude (this is a big advantage of being able to construct the tessellation geometrically, because a large, statistically reliable, sample of vertices is easily and quickly obtained).

Weinberg (1989) followed the same approach, distributing $32^{3}$ particles in a $64^{3}$ volume and choosing random locations for $N_{\mathrm{s}}=\mathrm{I} 6$ nuclei, representing the sites of the explosions that sweep up the surrounding material. Each particle is then projected radially outward from the nearest nucleus until it becomes equidistant from this and another one. This leaves all the particles on the walls of the tessellation, each wall having a uniform distribution of particles (but number density differing from wall to wall). Weinberg used this distribution to test the behaviour of his 'Gaussianization' initial density field reconstruction method on a model which violates the method's central assumption of Gaussian fluctuations and gravitational instability. The method was fairly successful in reproducing the density distribution, but showed clear differences between model and reconstructed correlation function. This showed that his method is potentially a powerful one for determining whether the observed galaxy distribution evolved from Gaussian initial density fluctuations by gravitational instability.

Finally, from an observational point of view, Pierre, Shaver \& Iovino (I988) and Pierre (I990) used the Voronoi tessellation to see whether one could say anything about the presence of cellular structures in the distribution of Lyman- $\alpha$ clouds, as derived from the absorption line spectra of quasars. The main conclusion was that any structure amongst the Ly- $\alpha$ absorbers is less pronounced than the void structure defined by the distribution of galaxies today.

\section{CONCLUSIONS}

The structure of our Universe on a scale of about 10-500 Mpc can be plausibly modelled by means of pressure-free Newtonian gravitational collapse. This mechanism produces a mass distribution which, asymptotically, is described by a statistical process known as Voronoi tessellation. Visual comparison between Voronoi foams and the observed distribution of galaxies shows a promising similarity. Detailed statistical study shows that the two-point correlation of Voronoi foam is practically indistinguishable from that which is obtained for the actual galaxy distribution. This holds in particular for the slope and the amplitude of the two-point correlation function of Abell clusters and of three-dimensional Voronoi vertices. 
Likewise, the cluster multiplicity function is properly reproduced. The most serious problem is that the CMBR fluctuations may be far too large.

\section{ACKNOWLEDGEMENTS}

It is a pleasure to thank J.H.Oort for his criticism and encouragement, D.Kendall for his interest in the advice about stochastic geometry, and V.Martínez, G.Rhee, P.Coles, D.Weinberg and T.Buchert for useful and encouraging discussions. We are grateful to J.D.Barrow for making available a copy of N.Ling's thesis, and to D.H.Weinberg for sending a copy of his thesis. We thank the referee for his incisive comments, which caused a substantial improvement of an earlier version of this article. We are especially indebted to B.Jones for his unflagging encouragement and enthusiasm, and for a careful appraisal of the manuscript.

\section{REFERENCES}

Abell, G.O., I958. Astrophys. J. Suppl., 3, 2 I I.

Bahcall, N.A., I988. Ann. Rev. Astron. Astrophys., 26, 631.

Bahcall, N.A. \& Soneira, R., I983. Astrophys. J., 270, 20.

Bahcall, N.A., Henriksen, M.J. \& Smith, T.E., 1989. Astrophys. J. (Letters), 346, L45.

Barrow, J.D., I988. In The Early Universe, Proceedings of the NATO Advanced Study Institute C 219, p. 125, eds Unruh, W.G. \& Semenoff, G.W., Reidel, Dordrecht.

Barrow, J.D., Bhavsar, S.P. \& Sonoda, D.H., 1985. Mon. Not. R. astr. Soc., $216,17$.

Barrow, J.D. \& Götz, 1989. Class. Quantum Grav., 6, 1253.

Barrow, J.D. \& Silk, J., I98I. Astrophys. J., 250, 432.

Batuski, D.J., Melott, A.L. \& Burns, J.O., I987. Astrophys. J., 322, 48.

Bertschinger, E., 1983. Astrophys. J., 268, I7.

Bertschinger, E., 1985. Astrophys. J. Suppl., 58, 39.

Bertschinger, E., Dekel, A., Faber, S.M. \& Dressler, A., I990. Astrophys. J., 364, 370.

Bhavsar, S.P. \& Barrow, J.D., I983. Mon. Not. R. astr. Soc., 205, 6I P.

Bhavsar, S.P. \& Ling, E.N., I988. Astrophys. J. (Letters), 33I, L63.

Binney, J. \& Tremaine, S., 1987. Galactic Dynamics, Princeton University Press, Princeton.

Bond, J.R., I990. In Frontiers in Physics: From Colliders to Cosmology, Proceedings of the Lake Louise Winter Institute, eds. Campbell, B. \& Khanna, F., World Scientific, Singapore. (in press).

Bouchet, F.R. \& Lachièze-Rey, M., 1986. Astrophys. J. (Letters), 302, L37.

Broadhurst, T.J., Ellis, R.S., Koo, D.C. \& Szalay, A.S., 1990. Nature, 343, 726.

Buchert, T., I989. Astron. Astrophys., 223, 9.

Centrella, J.M. \& Melott, A.L., 1983. Nature, 305, 196.

Centrella, J.M. Gallagher, J.S., Melott, A.L. \& Bushouse, H.A., I988. Astrophys. J., 333, 24. Chandrasekhar, S., 1969. Ellipsoidal Figures of Equilibrium, Yale University Press, New Haven.

Coleman, P.H., Pietronero, L. \& Sanders, R.H., 1988. Astron. Astrophys., 200, L32.

Coles, P., I990. Nature, 346, 446.

Coles, P. \& Barrow, J.D., I990. Mon. Not. R. astr. Soc., 244, 557.

Coles, P. \& Jones, B.J.T., I99I. Mon. Not. R. astr. Soc., 248, I.

Da Costa, L.N. \& Pellegrini, P.S., 1988. In: Large Scale Structure in the Universe, IAU Symposium I30, eds Audouze, J., Pelletan, M.C. \& Szalay, A., p. I2 I. Reidel, Dordrecht.

Davis, M., Huchra, J., Latham, D.W. \& Tonry, J., I982. Astrophys. J., 253, 423.

Davis, M. \& Peebles, P.J.E., I983. Astrophys. J., $267,465$.

Dekel, A., 1983. Astrophys. J., 264, 373.

Dekel, A., Bertschinger, E. \& Faber, S., I990. Astrophys. J., 364, 349.

Dekel, A., Blumenthal, G.R., Primack, J.R. \& Olivier, S., 1989. Astrophys. J. (Letters), 338, L5. Dekel, A. \& West, M., I985. Astrophys. J., 288, 4I I.

De Lapparent, V., Geller, M.J. \& Huchra, J.P., 1986. Astrophys. J. (Letters), 302, Li.

Dirichlet, G.L., I850. J. reine angew. Math., 40, 209. 
Dressler, A., Faber, S.M., Burstein, D., Davies, R.L., Lynden-Bell, D., Terlevich, R.J. \& Wegner, G., I987. Astrophys. J. (Letters), 313, L37.

Efstathiou, G., 1990. In Physics of the Early Universe, Proceedings of the Thirty-Sixth Scottish Universities Summer School in Physics 1989, p. 361, eds Peacock, J.A., Heavens, A.F. \& Davies, A.T., SUSSP Publications, Edinburgh.

Efstathiou, G. \& Silk, J., 1983. Fundam. Cosm. Phys., 9, I.

Einasto, J., 1978. In IAU Symposium No. 179, The Large Scale Structure of the Universe, p. 52, eds Longair, M.S. \& Einasto, J., Reidel, Dordrecht.

Einasto, J., Joêveer, M. \& Saar, E., I980. Mon. Not. R. astr. Soc., 193, 353.

Fillmore, J.A. \& Goldreich, P., 1984. Astrophys. J., 281, 9.

Fisher, J.R. \& Tully, R.B., 198I. Astrophys. J. Suppl., 47, I39.

Fujimoto, M., 1983. Publ. Astron. Soc. Japan, 35, I59.

Geller, M., I988. In Large Scale Structures in the Universe, I7th Saas-Fee course, eds Martinet, L. \& Mayor, M., Geneva Observatory, p. 69.

Geller, M. \& Huchra, J., 1989. Science, 246, 897.

Giovanelli, R., Haynes, M.P. \& Chincarini, G.L., I986. Astrophys. J., 300, 77.

Gott, J.R., Melott, A.L. \& Dickinson, M., I986. Astrophys. J., 306, 34I.

Gregory, S.A. \& Thompson, L.A., 1978. Astrophys. J., 222, 784.

Groth, E.J. \& Peebles, P.J.E., I977. Astrophys. J., 217, 385.

Gurbatov, S.N., Saichev, A.I. \& Shandarin, S.F., 1989. Mon. Not. R. astr. Soc., 236, 385.

Hausman, M.A., Olson, D.W. \& Roth, B.D, 1983. Astrophys. J., 270, 35 I.

Hoffman, G.L., Salpeter, E.E. \& Wasserman, I., I983. Astrophys. J., 268, 527.

Hoffman, Y. \& Shaham, J., 1982. Astrophys. J. (Letters), 262, L23.

Huchra, J.P., Davis, M., Latham, D. \& Tonry, L.J., 1983. Astrophys. J. Suppl., 52, 89.

Humason, M.L., Mayall, N.U. \& Sandage, A.R., I956. Astron, J., 6I, 97.

Icke, V., 1972. Formation of galaxies inside clusters, Ph.D. Thesis, Leiden.

Icke, V., I973. Astron. Astrophys., 27, I.

Icke, V., I984. Mon. Not. R. astr. Soc., 206, IP.

Icke, V. \& Poelman, Y., I99I. Preprint.

Icke, V. \& Van de Weygaert, R., 1987. Astron. Astrophys., I84, I6.

Ikeuchi, S., I98I. Pub. Astr. Soc. Japan, 33, 2 I I.

Ikeuchi, S., Tomisaka, K. \& Ostriker, J.P., I983. Astrophys. J., 265, 583.

Ikeuchi, S. \& Umemura, M., 1984. Prog. Theor. Phys., 72, 216.

Jones, B.J.T., 1976. Revs. Mod. Phys., 48, 107.

Jones, B.J.T., Martínez, V.J., Saar, E. \& Einasto, J., 1988. Astrophys. J. (Letters), 332, LI.

Johnson, W.A. \& Mehl, R.F., 1939. Trans. Am. Inst. Min. Metal Eng., 135, 4 I6.

Kaiser, N., 1984. Astrophys. J. (Letters), 284, L9

Kiang, T., 1966. Zeitschr. f. Astrophys., 64, 433.

Kirshner, R.P., Oemler, A., Schechter, P.L. \& Shectman, S.A., I98I. Astrophys. J. (Letters), 248, L57.

Kirshner, R.P., Oemler, A., Schechter, P.L. \& Shectman, S.A., 1987. Astrophys. J., 314, 493.

Klypin, A.A. \& Kopylov, A.I., 1983. Soviet Astr. Lett., 91, 267.

Klypin, A.A. \& Shandarin, S.F., I983. Mon. Not. R. astr. Soc., 204, 891.

Kofman, L.A., 1989. Cracow Summer School on Cosmology, August I988, Springer, Berlin.

Kofman, L.A. \& Shandarin, S.F., I988. Nature, 334, I 29.

Kofman, L.A., Pogosyan, D. \& Shandarin, S.F., 1990. Preprint.

Lake, K. \& Pim, R., 1985. Astrophys. J., 298, 439.

Lifschitz, E.M., 1946. J. Phys. U.S.S.R., I0, I16.

Limber, D.N., 1953. Astrophys. J., II7, I34.

Limber, D.N., 1954. Astrophys. J., I19, 655 .

Lin, C.C., Mestel, L. \& Shu, F.H., I965. Astrophys. J., I42, I431.

Ling, E.N., 1987. New statistical approaches to galaxy clustering, D.Phil. Thesis, University of Sussex.

Lynden-Bell, D., I964. Astrophys. J., I39, I195.

Lynden-Bell, D., Faber, S.M., Burstein, D., Davies, R.L., Dressler, A., Terlevich, R.J. \& Wegner, G., I988. Astrophys. J., 326, 19.

Maddox, S.J., Efstathiou, G., Sutherland, W.J. \& Loveday, J., 1990. Mon. Not. R. astr. Soc., 242, $43 \mathrm{P}$.

Maeda, K., Sasaki, M. \& Sato, M., 1982. Prog. Theor. Phys., 69, 89.

Maeda, K. \& Sato, H., 1983. Prog. Theor. Phys., 70, 772. 
Martínez, V.J. \& Jones, B.J.T., I990. Mon. Not. R. astr. Soc., 242, 517.

Martínez, V.J., Jones, B.J.T., Domínguez-Tenreiro, R. \& Van de Weygaert, R., 1990. Astrophys. J., 357, 50.

Matsuda, T. \& Shima, E., I984. Prog. Theor. Phys., 71, 855.

Maurogordato, S. \& Lachièze-Rey, M., 1987. Astrophys. J., 328, 50.

Melott, A.L.., 1983. Mon. Not. R. astr. Soc., 205, 637.

Melott, A.L., Weinberg, D.H. \& Gott, J.R., I988. Astrophys. J., 328, 50.

Meyering, J.L., 1953, Philips Res. Rept., 8, 270.

Miles, R.E., I970. Math. Biosci., 6, 85.

Møller, J., 1989. Adv. Appl. Appl. Prob., 21, 37.

Neymann, J. \& Scott, E.L., 1955. Astron. J., 60, 33.

Occhionero, F., Santangelo, P. \& Vittorio, N., I983. Astron. Astrophys., II7, 365.

Olivier, S., Blumenthal, G.R., Dekel, A., Primack, J.R. \& Stanhill, D., I990. Astrophys. J., 356, I.

Oort, J.H., 1970. Astron. Astrophys., 7, 38I.

Oort, J.H., 1983. Ann. Rev. Astron. Astrophys., 21, 373.

Ostriker, J.P. \& Cowie, L.L., I981. Astrophys. J. (Letters), 243, LI 27.

Ostriker, J.P. \& McKee, C.F., I988. Rev. Mod. Phys., 60, I.

Ostriker, J.P. \& Strassler, M., I989. Astrophys. J., 338, 579.

Peebles, P.J.E., 1980. The Large-Scale Structure of the Universe, Princeton University Press.

Peebles, P.J.E., I982. Astrophys. J., 257, 438.

Peebles, P.J.E. \& Silk, J., I990. Nature, 346, 233.

Pierre, M., 1990. Astron. Astrophys., 229, 7.

Pierre, M., Shaver, P. \& Iovino, A., I988. Astron. Astrophys. (Lett.), 197, L3.

Postman, M., Geller, M.J. \& Huchra, J.P., 1986. Astron. J., 9I, 267.

Pim, R. \& Lake, K., 1986. Astrophys. J., 304, 75.

Pim, R. \& Lake, K., I988. Astrophys. J., 330, 625.

Rood, H.J., 1988. Ann. Rev. Astron. Astrophys., 26, 245.

Rubin, V.C., I954. Proc. Nat. Acad. Sciences, 40, 54I.

Saarinen, S., Dekel, A. \& Carr, B., I987. Nature, 314, 598.

Sandage, A. \& Tammann, G.A., I98I. A Revised Shapley-Ames Catalog of Bright Galaxies,

Carnegie Inst. Washington, Publ. 633 .

Sato, H., 1982. Prog. Theor. Phys., 68, 236.

Sato, H. \& Maeda, K., I983. Prog. Theor. Phys., 70, I19.

Schwarz, J., Ostriker, J.P. \& Yahil, A., I975. Astrophys. J., 202, I.

Shandarin, S.F. \& Zel'dovich, Ya.B., 1989. Revs. Mod. Phys., 61, 185.

Shane, C.D. \& Wirtanen, C.A., I967. Publ. Lick Obs., 22, Part I.

Shapley, H. \& Ames, A., 1932. Harvard Obs. Ann., 88, 43.

Stoyan, D., Kendall, W.S. \& Mecke, J., 1987. Stochastic Geometry and Its Applications, Akademie-Verlag, Berlin.

Strauss, M. \& Davis, M., I989. In Large Scale Motions in the Universe, Pont. Acad. Sci. Study

Week No. 27, p. 256, eds Rubin, V. \& Coyne, G., Princeton University Press.

Sutherland, W.J., I988. Mon. Not. R. astr. Soc., 234, I59.

Suto, Y., Sato, K. \& Sato, H., 1984. Prog. Theor. Phys., 71, 938.

Szalay, A., 1988. In Large Scale Structures in the Universe, 17 th Saas-Fee course, p. 173, eds

Martinet, L. \& Mayor, M., Geneva Observatory.

Toomre, A. \& Toomre, J., 1972. Astrophys. J., 178, 623.

Totsuji, H. \& Kihara, T., 1969. Publ. Astron. Soc. Japan, 2I, 221.

Tully, R.B., I982. Astrophys. J., 257, 389.

Van de Weygaert, R., I99I. Mon. Not. R. astr. Soc., (in press).

Van de Weygaert, R. \& Icke, V., I989. Astron. Astrophys., 213, I.

Van de Weygaert, R., Jones, B.J.T. \& Martínez, V.J., I99I. Phys. Lett. A. (in press).

Voronoi, G., 1908. J. reine angew. Math., 134, 198.

Weinberg, D.H., 1989. Reconstructing primordial density fluctuations, Ph.D. Thesis, Princeton University.

Weinberg, D.H., Gott, J.R. \& Melott, A.L., I987. Astrophys. J., 32I, 2.

Weinberg, D.H., Ostriker, J.P. \& Dekel, A., I989. Astrophys. J., 336, I.

Weinberg, D.H. \& Gunn, J.E., 1990. Astrophys. J. (Letters), 352, L25.

White, S.D.M., I979. Mon. Not. R. astr. Soc., I86, I45.

White, S.D.M. \& Silk, J., 1979. Astrophys. J., 23I, I. 
White, S.D.M., Frenk, C.S., Davis, M. \& Efstathiou, G., I987. Astrophys. J., 3I3, 505.

Yahil, A., 1989. In Large Scale Motions in the Universe, Pont. Acad. Sci. Study Week, No. 27, p. 129, eds Rubin, V. \& Coyne, G., Princeton University Press.

Yoshioka, S. \& Ikeuchi, S., I989. Astrophys. J., 34I, I6.

Zel'dovich, Ya.B., I965. Soviet Astron., 8, 700.

Zel'dovich, Ya.B., I970. Astron. Astrophys., 5, 84.

Zel'dovich, Ya.B., I978. In IAU Symposium No. 79, The Large Scale Structure of the Universe, p. 409, eds Longair, M.S. \& Einasto, J., Reidel, Dordrecht.

Zel'dovich, Ya.B., Einasto, J. \& Shandarin, S.F., I982. Nature, 300, 407.

Zeng, N. \& White, S.D.M., I990. Preprint. 